\title{
SOX5 promotes breast cancer proliferation and invasion by transactivation of EZH2
}

\author{
CHUNTAO SUN $^{1}$, YUNQING BAN ${ }^{2}$, KAI WANG $^{3}$, YANMING SUN $^{1}$ and ZHIHUA ZHAO ${ }^{4}$ \\ ${ }^{1}$ Department of Interventional Radiology, Weifang City People's Hospital, Weifang, Shandong 261041; ${ }^{2}$ Imaging Center, \\ The 5th Affiliated Hospital of Xinjiang Medical University, Urumqi, Xinjiang 830011; Departments of ${ }^{3}$ Breast \\ Surgery and ${ }^{4}$ Nuclear Medicine, Weifang City People's Hospital, Weifang, Shandong 261041, P.R. China
}

Received June 21, 2017; Accepted August 15, 2018

DOI: $10.3892 / \mathrm{ol} .2019 .9914$

\begin{abstract}
Sex determining region Y-box protein 5 (SOX5) is a transcriptional factor and serves important roles in various cancer types; however, the pathological role of SOX5 in patients with breast cancer remains unclear. In the present study, the expression and potential role of SOX 5 in patients with breast cancer and in breast cancer cells was investigated. The data indicated that SOX5 was highly expressed in breast cancer tissues compared with adjacent healthy tissues, and overexpression of SOX5 was associated with a reduced overall survival rate in patients with breast cancer. Gain and loss of function studies with MTT, colony formation, wound healing and Matrigel invasion assays demonstrated that SOX5 significantly promoted breast cancer cell proliferation and invasion. The chromatin immunoprecipitation (ChIP) assay sequence, quantitative ChIP and luciferase reporter assays were used to identify enhancer of zeste 2 polycomb repressive complex 2 subunit (EZH2) as a downstream target gene of SOX5. Furthermore, it was determined that ectopic expression of SOX 5 increased EZH2 expression at the mRNA and protein level, while the knockdown of SOX 5 decreased EZH 2 expression. Additionally, the biological effect of SOX5 was investigated, and it was determined to be dependent on the regulation of EZH2 expression. The present results may provide important insights into the biological significance of SOX5 serving as a candidate therapeutic target in breast cancer progression.
\end{abstract}

\section{Introduction}

Breast cancer is one of the most common cancer types among females worldwide in 2014, which cause cancer-associated

Correspondence to: Mr. Kai Wang, Department of Breast Surgery, Weifang City People's Hospital, 151 Guangwen Street, Weifang, Shandong 261041, P.R. China

E-mail: dlzfbt@163.com

Key words: sex determining region Y-box protein 5, enhancer of zeste 2 polycomb repressive complex 2 subunit, breast cancer, proliferation, invasion mortalities globally $(1,2)$. In China, breast cancer remains the most common type of neoplasm in 2014 (3). With the development of medical technologies over the past 20 years, including surgery, radiotherapy and chemotherapy, the diagnosis and treatment of breast cancer have continuously improved $(4,5)$. Metastasis and recurrence remain the major causes of high mortality rates of patients with breast cancer (6); therefore, understanding the mechanisms and investigating novel biomarkers, which are responsible for unfavorable progression, is important. Furthermore, the identification of novel therapeutic targets for breast cancer treatment is essential.

Sex determining region Y-box protein 5 (SOX5) is a member of the SOX family, which was identified based on the conserved homology of the high-mobility group DNA-binding motif (7). It has been reported that SOX5 is involved in the regulation of embryonic development (8), and is associated with various cancer types, including prostate cancer (9), glioblastoma (10), hepatocellular carcinoma (11), osteosarcoma (12) and nasopharyngeal carcinoma (13). In 2014, Pei et al (14) reported that in breast cancer, SOX5 induces epithelial-mesenchymal transition (EMT) by transactivation of Twist1 expression. However, the expression and the precise regulatory mechanism underlying the biological function of SOX5 in breast cancer remain unclear.

\section{Patients and methods}

Cell culture and reagents. The normal breast tissue cell line, MCF-10A, and the MCF7, T47D and MDA-MB-231 breast cancer cell lines were obtained from the American Type Culture Collection (Manassas, VA, USA). MCF-7 and T47D cells were cultured in Dulbecco's modified Eagle's medium (DMEM; Gibco; Thermo Fisher Scientific, Inc., Waltham, MA, USA), and MDA-MB-231 cells were cultured in RPMI-1640 supplemented with $10 \%$ fetal bovine serum (FBS; both Invitrogen; Thermo Fisher Scientific, Inc.). MCF-10A cells were cultured in DMEM-F12 supplemented with 5\% horse serum (Invitrogen; Thermo Fisher Scientific, Inc.). The cells were incubated in an atmosphere containing $5 \% \mathrm{CO}_{2}$ at $37^{\circ} \mathrm{C}$. Lipofectamine ${ }^{\circledR} 2000$ (Invitrogen; Thermo Fisher Scientific, Inc.) was used for transfection to transfected into MCF-7 or MDA-MB-231 cells. The relative small interfering (si)RNAs targeting SOX5 (si-SOX5-1 and si-SOX5-2) or enhancer of zeste 2 polycomb repressive 
complex 2 subunit (EZH2) or negative control and G418 were purchased from Sigma-Aldrich (Merck KGaA, Darmstadt, Germany). SOX5 and vector plasmid were purchased from Genepharma (Shanghai, China). The $70 \%$ confluence of MCF-7 or MDA-MB-231 cells were achieved overnight prior to transfection. In each group, $2 \mu \mathrm{g}$ oligonucleotide were used for transfection. At $48 \mathrm{~h}$ following transfection, the cells were harvested for experimentation.

Patients. The present study was approved by the Research Ethics Committee of Weifang People's Hospital (Weifang, China). All patients provided written informed consent. A total of 58 pairs of breast cancer tissues from female patients aged from 40-55 years old and relative adjacent healthy mammary tissues were collected between May 2010 and January 2013. The fresh specimens were frozen immediately at $-80^{\circ} \mathrm{C}$ in liquid nitrogen for reverse transcription-quantitative polymerase chain reaction (RT-qPCR) use. Patients who received tumor-specific therapy prior to diagnosis were excluded. The pathological information was retrieved by the Pathology Department of Weifang People's Hospital. Overall survival times were calculated as the duration between the date of diagnosis and date of cancer-associated mortality in the follow-up period.

RNA extraction and RT-qPCR analysis. Total RNA was extracted from cells or tissues using TRIzol ${ }^{\circledR}$ reagent (Invitrogen; Thermo Fisher Scientific, Inc.), according to the manufacturer's protocol. First-strand complementary DNA was synthesized using SuperScript II Reverse Transcriptase kit (Invitrogen; Thermo Fisher Scientific, Inc.). qPCR was performed using the Fast SYBR ${ }^{\circledR}$ Green Master mix system (Roche Applied Science, Penzberg, Germany) on an ABI 7500 system (Applied Biosystems; Thermo Fisher Scientific, Inc.). The qPCR reaction was subsequently performed according to the following conditions: Initial step, $95^{\circ} \mathrm{C}$ for $5 \mathrm{~min}$; second step, $95^{\circ} \mathrm{C}$ for $10 \mathrm{sec}$, $60^{\circ} \mathrm{C}$ for $30 \mathrm{sec}$ and $72^{\circ} \mathrm{C}$ for $10 \mathrm{sec}$ for a total of 35 cycles. The primers used were as follows: EZH2 forward, 5'-TTTCCA ACACAAGTCATCCC-3', and reverse, 5'-ATAAACCCACAT TCTCTATCCC-3'; GAPDH forward, 5'-CCGTCTAGAAAA ACCTGCC-3', and reverse, 5'-GCCAAATTCGTTGTCATA CC-3'. The relative $\mathrm{mRNA}$ level was calculated using the $2^{-\Delta \Delta \mathrm{Cq}}$ method and normalized to GAPDH (15). The experiment was performed in triplicate.

Western blot analysis. MCF-7 or MDA-MB-231 cells were harvested and protein was extracted using radioimmunoprecipitation buffer $(50 \mathrm{mM}$ tris- $\mathrm{HCl} \mathrm{pH} 7.4,1 \%$ Triton X-100, $1 \%$ sodium deoxycholate, $150 \mathrm{mM} \mathrm{NaCl}$ and $0.1 \%$ SDS). The Bradford assay reagent (Thermo Fisher Scientific, Inc.) was then used to determine the protein concentration in the lysates. Equal amounts of protein $(30 \mu \mathrm{g})$ were separated by $10 \%$ SDS-PAGE gel, and then transferred to a polyvinylidene fluoride membrane (EMD Millipore, Billerica, MA, USA). The membranes were blocked in 5\% non-fat milk in PBS containing $0.5 \%$ Tween-20 at room temperature for $1 \mathrm{~h}$ and incubated with the primary antibodies overnight at $4^{\circ} \mathrm{C}$, and then washed three times with washing buffer Tris-buffered saline Tween-20 (Sigma-Aldrich, Merck KGaA). Horseradish peroxidase-conjugated anti-rabbit (sc-2357; 1:3,000) or anti-mouse (sc-2789, 1:3,000; both Santa Cruz Biotechnology, Inc., Dallas, TX, USA) antibodies were used as the secondary antibodies at room temperature for $1 \mathrm{~h}$. The signal was visualized using enhanced chemiluminescence reagents (Pierce; Thermo Fisher Scientific, Inc.). The primary antibodies used were as follows: SOX5 (ab94396; 1:1,000), EZH2 (ab186006; 1:1,000; both Abcam). $\beta$-actin (sc-47778; 1:1,000; Santa Cruz Biotechnology, Inc.) was used as the control.

Chromatin immunoprecipitation assay (ChIP). The ChIP assay was performed using Chip-IT Express kit (Active Motif; Carlsbad, CA, USA), according to manufacturer's protocols. The PCR products were resolved using a ABI 7500 system. PCR was performed with $5 \mu 1$ of the immunoprecipitated target DNA, $1 \mu \mathrm{l}$ primers and $9 \mu \mathrm{l}$ mixture $(1 \mu \mathrm{l}$ enzyme, $2 \mu \mathrm{l}$ dNTP and $6 \mu$ SYBR green solution buffer all were included in the ChIP-IT kit.

ChIP sequencing. For ChIP sequencing, the DNA was purified with the Qiagen PCR purification kit. In-depth whole genome DNA sequencing was performed by the CapitalBio Corporation. The raw sequencing image data were examined by the Illumina analysis pipeline, aligned to the unmasked human reference genome (NCBI v36, hg18) using ELAND (Illumina), and further analyzed by MACS (Model-based Analysis for ChIP-Seq).

Luciferase report assay. A luciferase report assay was performed using a dual luciferase assay kit according to the manufacturer's protocol (Promega Corporation, Madison, WI, USA). The EZH2 promoter was cloned into the luciferase reporter pGL3-basic vector plasmid, which was part of the kit. A total of $5 \times 10^{4}$ cells-well were cultured in DMEM at $37^{\circ} \mathrm{C}$ in 24 -well plates for $48 \mathrm{~h}$. The report plasmid was transfected into the cells with the relative plasmid SOX5 or shRNA targeting SOX5 or a negative control plasmid. After $24 \mathrm{~h}$ transfection, the luciferase activities were measured according to the aforementioned kit. The result was normalized to Renilla. The transfections were performed in triplicate.

Cell proliferation assay. For the MTT assay, $5 \times 10^{3}$ cells were seeded into 96-well plates with $100 \mu \mathrm{l}$ culture medium (DMEM; Gibco; Thermo Fisher Scientific, Inc.) and cultured at $37^{\circ} \mathrm{C}$ for different periods of time at $0,24,48$ and $72 \mathrm{~h}$. A total of $10 \mu \mathrm{l} 5 \mathrm{mg}-\mathrm{ml}$ MTT reagent (Beyotime Institute of Biotechnology, Shanghai, China) was added into each well and the culture was continued for $4 \mathrm{~h}$. Subsequently, $100 \mu \mathrm{l}$ dimethyl sulfoxide was used to replace the medium. After 30 min of incubation, the absorbance at $570 \mathrm{~nm}$ wavelength was measured on a SpectraMax 190 microplate reader (Bio-Rad Laboratories, Inc., Hercules, CA, USA) and cell growth curves were determined. Experiments were performed in triplicate independently.

Colony formation assay. For the colony formation assay, the cells were seeded into 6 -well plates with $1 \times 10^{3}$ cells-well. Fresh culture medium (DMEM; Gibco; Thermo Fisher Scientific, Inc.) was replaced every 3 days and cultured at $37^{\circ} \mathrm{C}$ in an atmosphere containing $5 \% \mathrm{CO}_{2}$ for 2 weeks, to form colonies. Subsequently, the cells were fixed with $70 \%$ methanol at room temperature for 30 mins and stained with $5 \%$ crystal violet at room temperature for 10 mins. The colonies containing $>50$ cells were counted under a Leica DMI 3000B inverted 

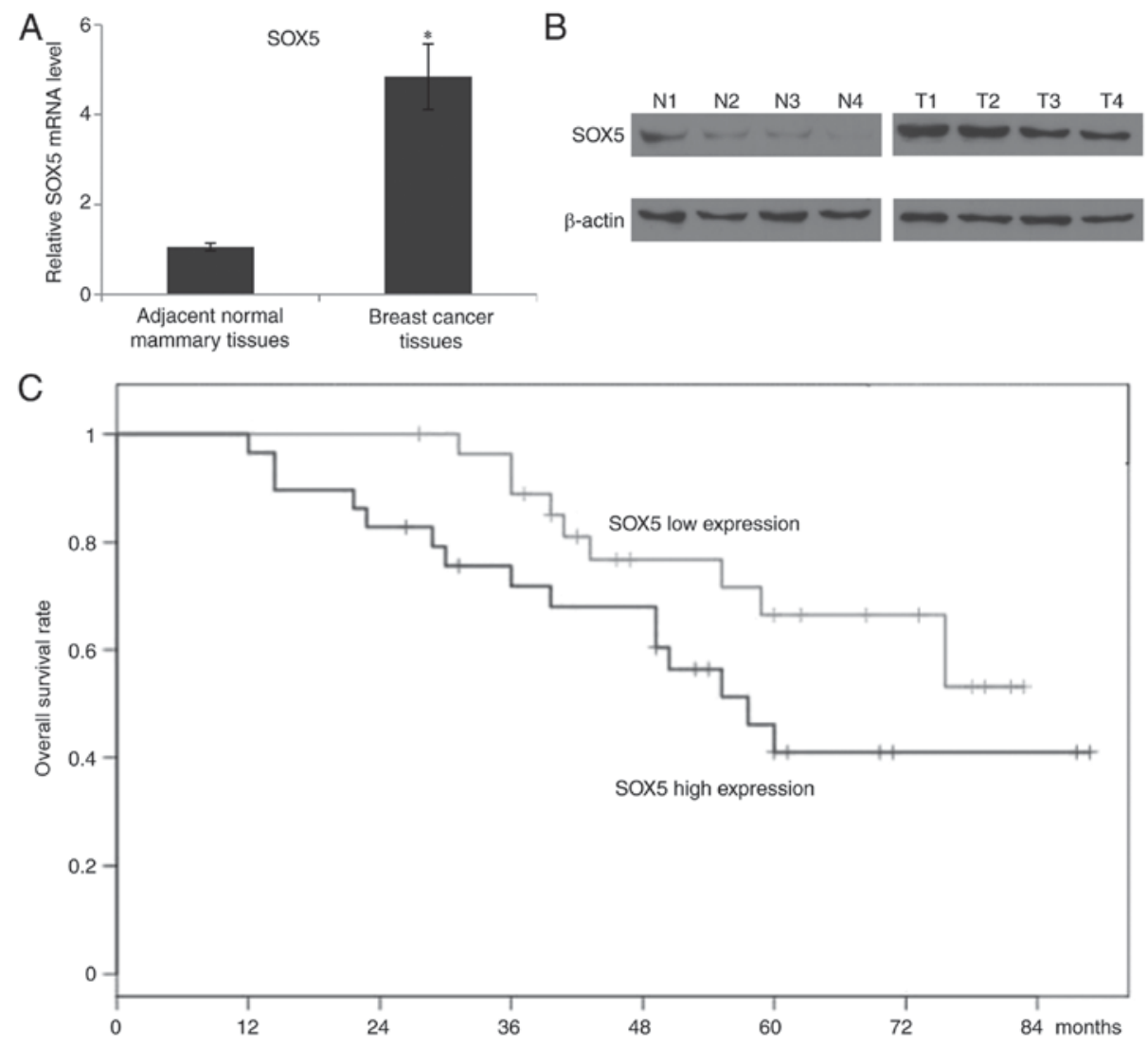

Figure 1. SOX5 is frequently upregulated in breast cancer tissues and associated with reduced overall survival rate. (A) The mRNA expression of SOX5 was measured using reverse transcription-quantitative polymerase chain reaction in breast cancer tissues, compared with adjacent healthy mammary tissues. All the experiments were repeated $\geq 3$ times and the data are presented as the mean \pm standard deviation. ${ }^{*}<<0.05$ compared with healthy mammary tissues. (B) The protein expression of SOX 5 was detected in 4 pairs of breast cancer tissues, compared with the relative adjacent healthy mammary tissues, and $\beta$-actin was used as the control. (C) Kaplan-Meier analysis was used to demonstrate that the increased SOX5 expression predicted an unfavorable overall survival rate in patients with breast cancer. SOX5, sex determining region Y-box protein 5.

microscope (Leica Microsystems, Inc., Buffalo Grove, IL, USA) at magnification, $\mathrm{x} 40$.

Scratch assay. Wound healing was used to observe the migration ability of breast cancer cells. A total of $5 \times 10^{4}$ cells were plated in 6-well plates and cultured until $95 \%$ confluency. A plastic $20 \mu \mathrm{l}$ pipette tip was used to scratch a vertical wound. Detached cells were removed and phase contrast images of the scratched fields were captured at 0 and $24 \mathrm{~h}$. In each group, at least three scratched fields were recorded using an upright light microscope at magnification, x20 (Leica DM4B; Leica Microsystems, Shanghai, China).

Invasion assay. A Matrigel assay was performed to investigate the invasion ability. Transwell chambers $(8-\mu \mathrm{m}$ pore size) were coated with 1 mg-ml Matrigel (both BD Biosciences, San Jose, CA, USA). Cells were seeded into $0.2 \mathrm{ml}$ serum-free medium (DMEM; Gibco; Thermo Fisher Scientific, Inc.) at a density of $1 \times 10^{4}$ cells-well and placed on the top chamber of each insert. The lower chamber was filled with $600 \mu 1$ medium (DMEM; Gibco; Thermo Fisher Scientific, Inc.) with $10 \%$ FBS. After $24 \mathrm{~h}$ of incubation, cells on the surface were wiped off by mechanical scraping, and the migrant cells attached to the lower surface were fixed with $10 \%$ methanol for $30 \mathrm{~min}$ at room temperature. Following staining with $5 \%$ crystal violet at room temperature for $20 \mathrm{~min}$, the cells were visualized and counted under a Leica DMI 3000B inverted microscope at magnification, $x 40$. A total of three different fields of view in each group were counted.

Statistical analysis. SPSS 19.0 (IBM Corp., Armonk, NY, USA) was used for statistical analysis. All data were expressed as the mean \pm standard deviation following $\geq 3$ independent experiments. $\mathrm{P}<0.05$ was considered to indicate a statistically significant difference. Kaplan-Meier analysis followed by the log-rank test was used to analyze the association between SOX5 expression and the overall survival rate. Significant differences between two groups were determined with a Student's t-test. One-way analysis of variance followed by Tukey's test was used to analyze the differences between multiple groups to compare values of test and control samples.

\section{Results}

SOX5 is frequently upregulated in breast cancer tissues and associated with a reduced overall survival rate. In order to identify the role of SOX5 in breast cancer, the expression of SOX5 in 58 pairs of matched breast cancer and adjacent healthy mammary tissues was investigated using RT-qPCR assays. Compared with the adjacent healthy tissues, significantly increased SOX5 mRNA expression levels were determined in breast cancer tissues (Fig. 1A). Furthermore, four pairs 
A

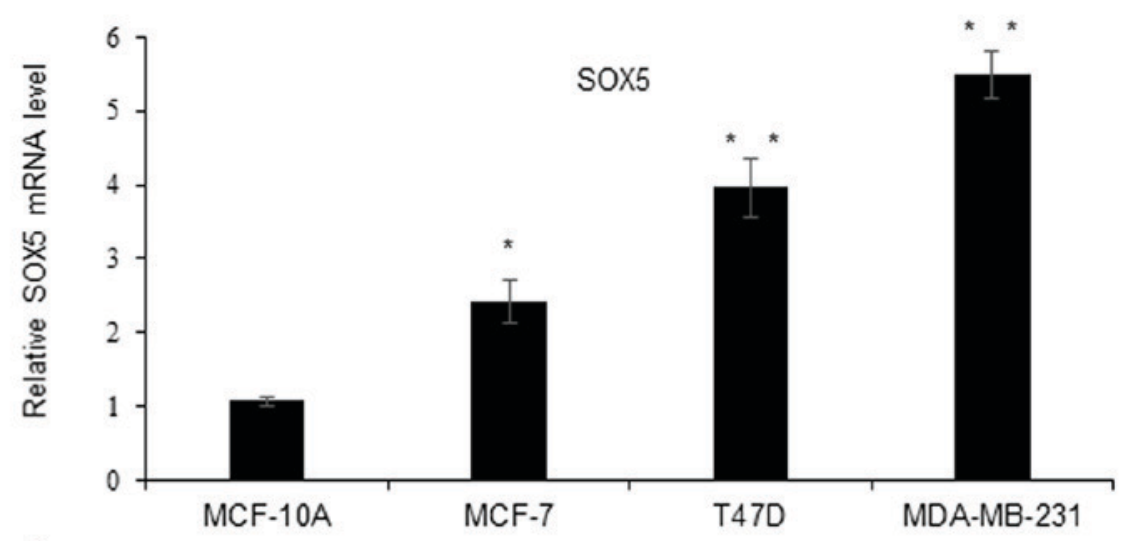

B
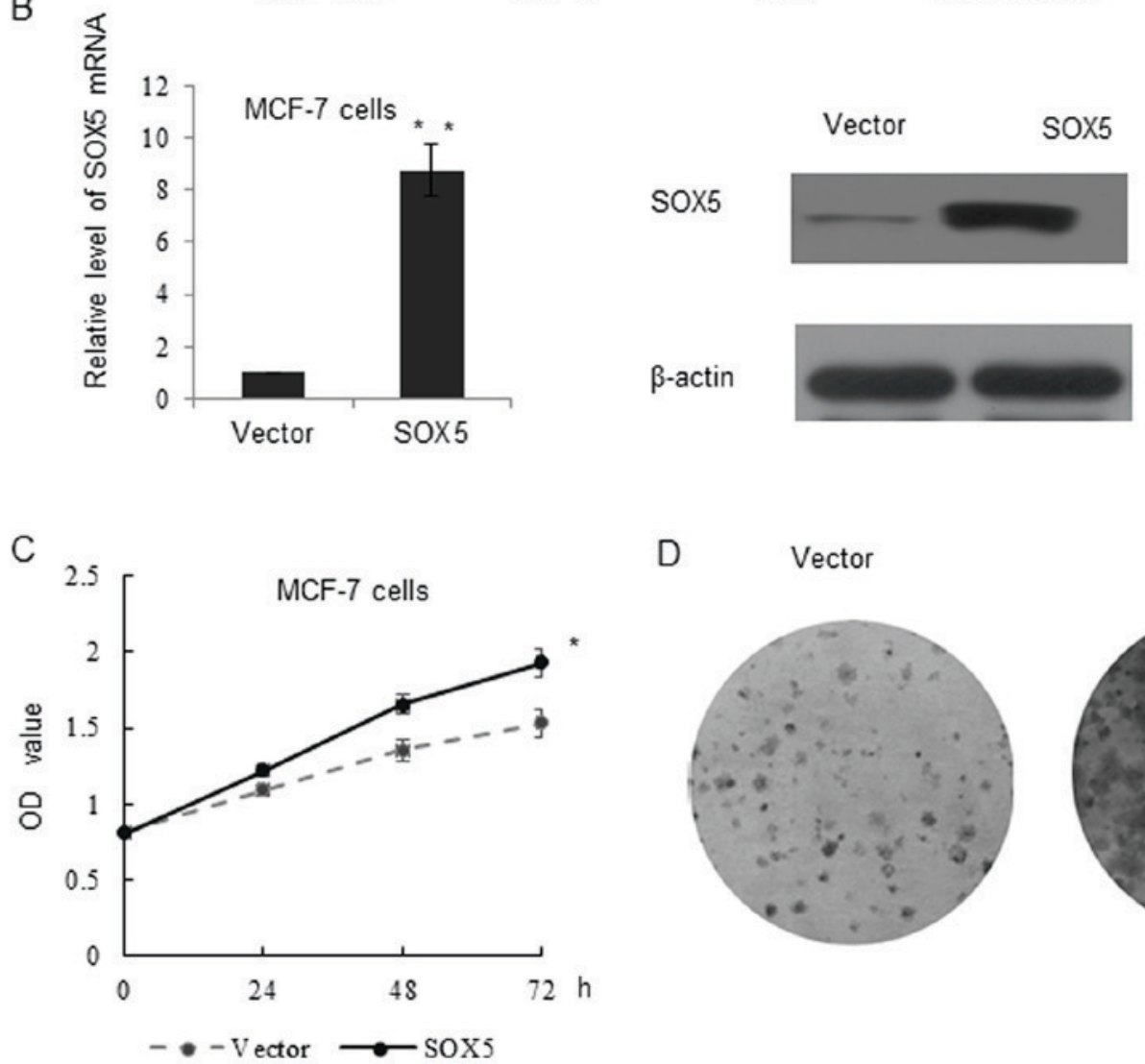

D Vector

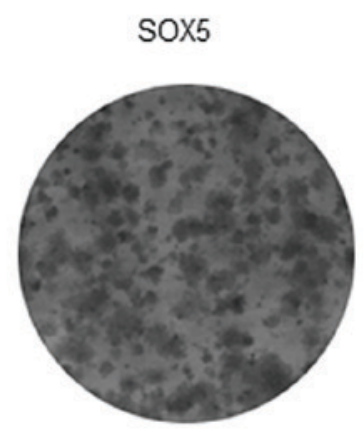

Figure 2. SOX5 promotes breast cancer cells proliferation in vitro. (A) The mRNA expression of SOX5 was measured using RT-qPCR in MCF-10A cells and breast cancer cells, including MCF-7, T47D and MDA-MB-231 cells. Data are presented as the mean \pm standard deviation. (B) RT-qPCR and western blotting analysis was used to examine SOX5 expression in MCF-7 cells transfected with the lentivirus expressing SOX5 or empty vector cells. (C) MTT analysis was performed in MCF-7 cells transfected with the lentivirus expressing SOX5 or a vector, and the results were detected at 0, 24, 48 and $72 \mathrm{~h}$. (D) Colony formation analysis was performed in MCF-7 cells transfected with lentivirus expressing SOX5 or a vector. "P<0.05 and ** P $<0.01$ compared with MCF-10A cells. SOX5, sex determining region Y-box protein 5; OD, optical density; RT-qPCR, reverse transcription-quantitative polymerase chain reaction.

of breast cancer and relative healthy mammary tissues were selected to detect the protein expression of SOX5. As depicted in Fig. 1B, western blotting demonstrated that SOX5 was notably overexpressed in breast cancer tissues compared with healthy breast tissue. Kaplan-Meier estimator analysis with the log-rank test was used to investigate the prognostic significance of SOX5 in patients with breast cancer. It was determined that an increased expression of SOX5 was significantly associated with a reduced overall survival rate $(\mathrm{P}=0.00213)$ (Fig. 1C).

SOX5 promotes breast cancer cell proliferation in vitro. In order to examine the role of SOX5 in breast cancer progression,
RT-qPCR was performed to investigate the expression of SOX5 in MCF-10A, MCF-7, T47D and MDA-MB-231 cells. Compared with the normal breast cancer cell line MCF-10A, the SOX5 mRNA level was significantly increased in the breast cancer cell lines. Additionally, the triple-negative cell line MDA-MB-231 exhibited the highest SOX5 expression level among all cell lines (Fig. 2A). Therefore, the MDA-MB-231 cell line was selected to perform the loss of function assay, while the MCF-7 cell line was selected for the gain of function assay. As depicted in Fig. 2B, stable transfection of SOX5 lentivirus was obtained following G418 selection and confirmed using RT-qPCR and western 

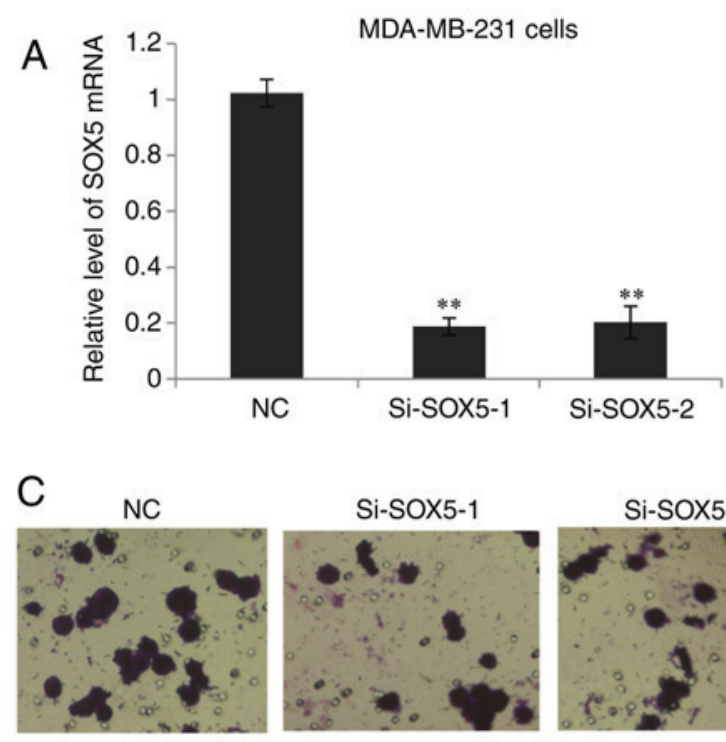

D
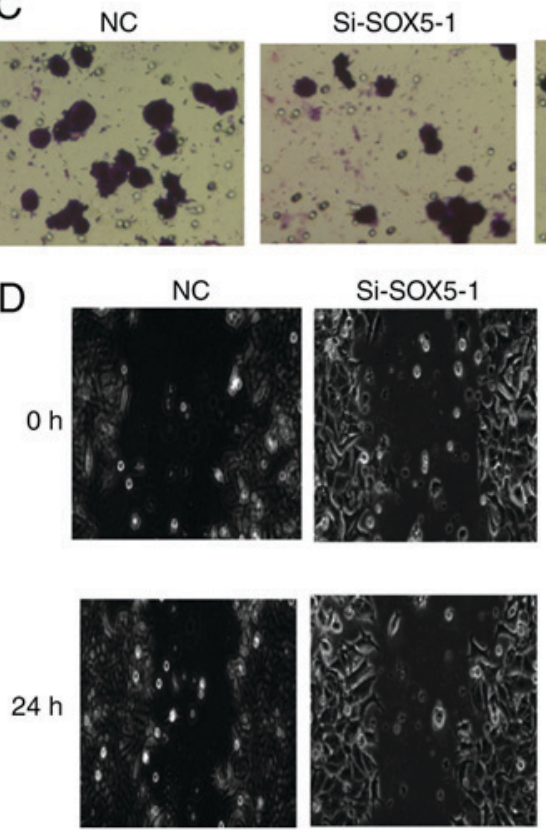

Si-SOX5-2

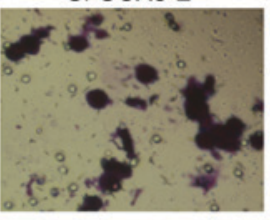

Si-SOX5-2
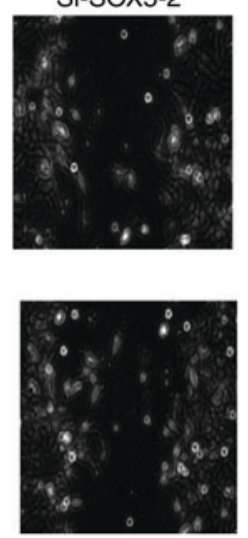

B

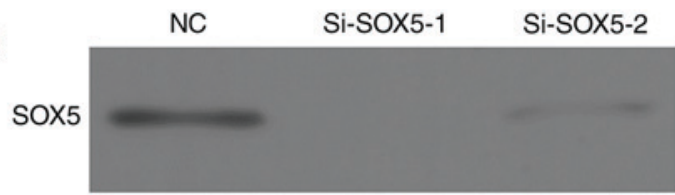

$\beta$-actin
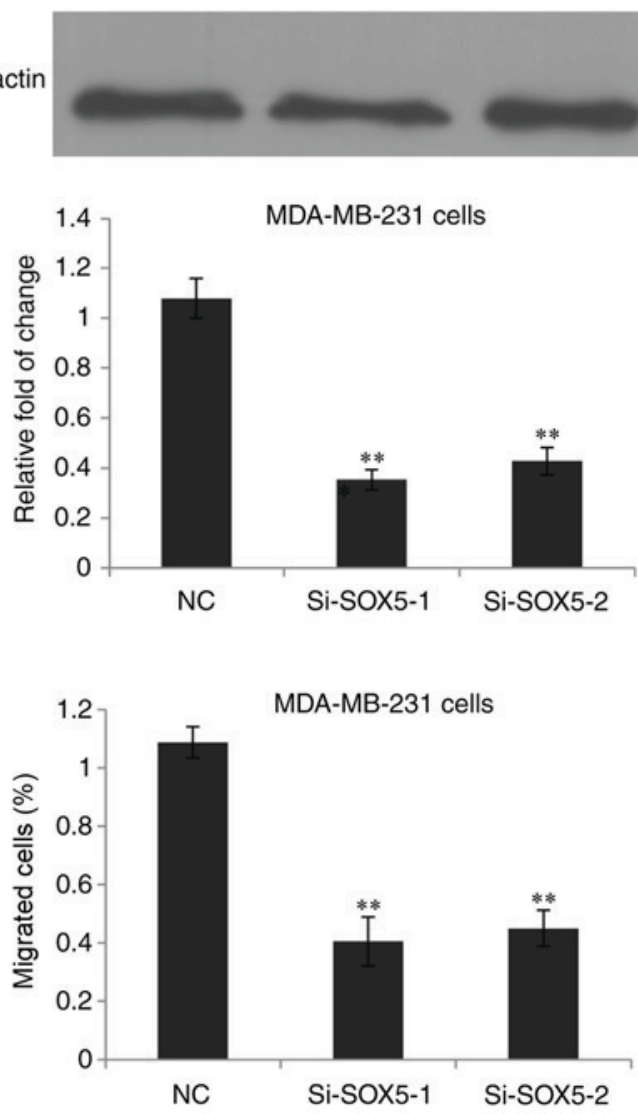

Figure 3. SOX5 enhances breast cancer cells invasion in vitro. (A) RT-qPCR analysis of SOX5 expression in MDA-MB-231 cells transfected with SOX5 siRNA (si-SOX5-1 and si-SOX5-2) as well as si-control. (B) Western blotting analysis of SOX5 expression in MDA-MB-231 cells transfected with SOX5 siRNA (si-SOX5-1 and si-SOX5-2) as well as si-control. (C) Matrigel analysis was performed in MDA-MB-231 cells of the si-SOX5-1 and si-SOX5-2 transfection groups, as well as the si-control group. (D) A wound-healing assay was used to evaluate the effect of SOX5 expression on MDA-MB-231 cell motility. Student's t-test was performed to evaluate the statistical significance, ${ }^{* *} \mathrm{P}<0.01$ compared with the si-NC group. Magnification, x100. SOX5, sex determining region Y-box protein 5; RT-qPCR, reverse transcription-quantitative polymerase chain reaction; NC, negative control; siRNA, small interfering RNA.

blotting. An MTT assay was performed, which indicated that cells overexpressing SOX5 proliferated significantly faster, compared with vector control cells (Fig. 2C). The colony formation assay demonstrated that SOX 5 formed larger and an increased number of colonies, compared with the vector control group (Fig. 2D).

SOX 5 enhances breast cancer cell invasion in vitro. To further investigate the role of SOX 5 in breast cancer cell invasion, SOX5 expression was silenced in MDA-MB-231 cells using two different siRNAs. Successful depletion of SOX5 expression was confirmed at the mRNA (Fig. 3A) and protein levels (Fig. 3B). As expected, inhibition of SOX5 significantly impeded the MDA-MB-231 cell invasion ability compared with the negative control group (Fig. 3C). Subsequently, a scratch assay was performed to assess the role of SOX5 in the migration of breast cancer cells. As depicted in Fig. 3D, the scratch assay revealed that SOX5 knock down significantly reduced the migratory ability of MDA-MB-231 cells. Therefore, these data indicated that
SOX5 exhibited the ability to promote MDA-MB-231 cell invasion and migration in vitro.

Identification of EZH2 as a downstream target gene of SOX5. Subsequently, the potential downstream molecule regulated by SOX5 was identified. A ChIP sequence (ChIP-seq) assay was performed. The ChIP-seq peak distribution is depicted in Fig. 4A, and $17.5 \%$ promoters were identified to be targeted by SOX5. To further validate the ChIP-seq results, a qChIP assay and the binding between SOX5 and the EZH2 promoter was demonstrated to be the most significantly enriched among the 10 genes selected (Fig. 4B). To investigate the SOX5-regulated EZH2 promoter activity, a luciferase report assay was performed. The EZH2 promoter reporter or EZH2 binding site mutant promoter reporter was transiently transfected into MCF-7 cells with pcDNA3.1-SOX5 or a vector. As depicted in Fig. 4C, SOX5 significantly activated EZH2 wild type promoter activity, but not the EZH2 mutant reporter activity. No significant changes in EZH2 promoter activity 
A

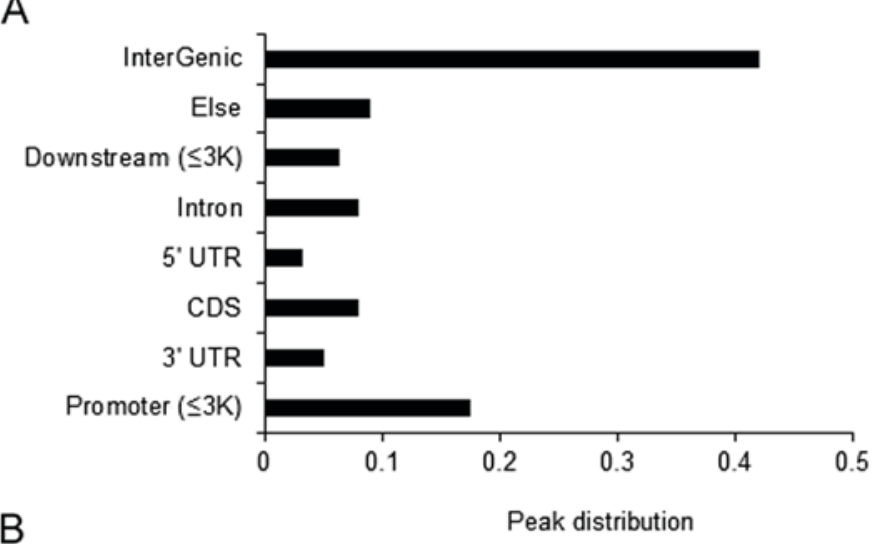

B

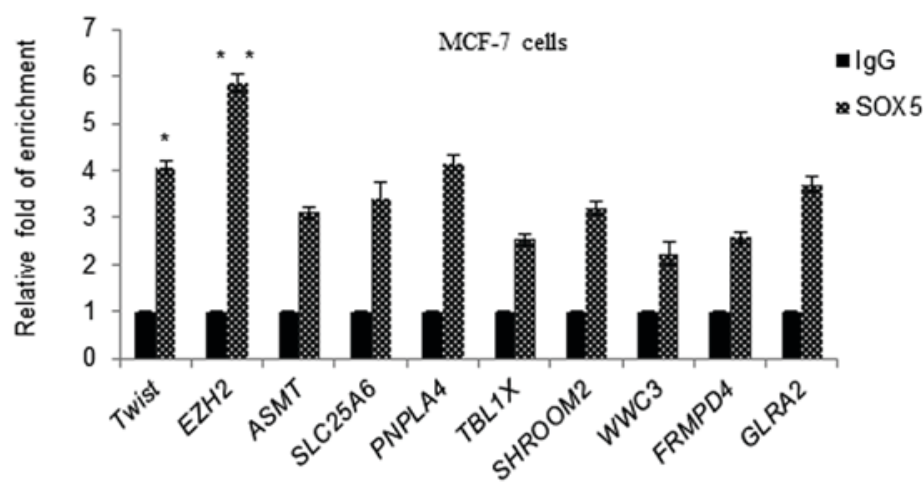

C

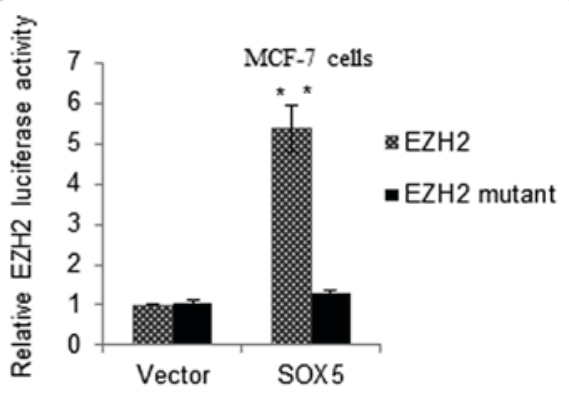

D

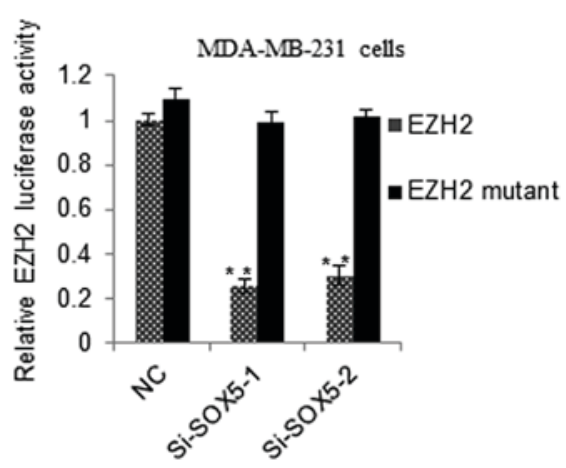

Figure 4. Identification of EZH2 as a downstream target gene of SOX5. (A) A ChIP-seq assay was performed in MCF-7 cells with a SOX5 antibody or a normal IgG, as a negative control, and the peak distributions were depicted. (B) A qChIP experiment was performed in MCF-7 or MDA-MB-231 cells, and the enrichments on the promoter of EZH2 were detected. Each bar indicated the mean \pm standard deviation of 3 independent experiments. " $\mathrm{P}<0.05$, ${ }^{* *} \mathrm{P}<0.01$ vs. IgG. (C) MCF-7 cells were transfected with EZH2 promoter reporter or EZH2 binding site mutant promoter reporter, and pcDNA3.1-SOX5 or vector. Luciferase activity was measured and normalized to Renilla. Experiments were repeated 3 times. ${ }^{* *} \mathrm{P}<0.01$ vs. Vector. (D) MDA-MB-231 cells were transfected with EZH2 promoter reporter or EZH2 binding site mutant promoter reporter, and si-SOX5 or si-control. Luciferase activity was measured and normalized to Renilla. Experiments were repeated 3 times. ${ }^{* *} \mathrm{P}<0.01$ vs. NC. SOX5, sex determining region Y-box protein 5; NC, negative control; siRNA, small interfering RNA; EZH2, enhancer of zeste 2 polycomb repressive complex 2 subunit; ChIP, chromatin immunoprecipitation; UTR, untranslated region; CDS, coding sequence; ASMT, acetylserotonin O-methyltransferase; SLC25A6, solute carrier family 25 member 6; PNPLA4, patatin like phospholipase domain containing 4; TBL1X, transducing $\beta$ like 1 X-linked; FRMPD4, FERM and PDZ domain containing 4; GLRA2, glycine receptor $\alpha 2$.

were observed in the vector control group. However, in MDA-MB-231 cells transfected with the EZH2 promoter reporter or EZH2 binding site mutant promoter reporter with si-SOX 5 or si-control, it was observed that si-SOX5 significantly repressed EZH2 promoter activity, but not the mutant promoter activity (Fig. 4D).

SOX 5 induces breast cancer cell proliferation and invasion by modulating EZH2. EZH2 is the catalytic subunit of polycomb repressive complex 2, and EZH2 had been demonstrated to serve a role in breast tumor initiation and progression (16-18).
Therefore, we hypothesized that the modulation of EZH2 was involved with SOX5, increasing breast cancer cell proliferation and invasion. As depicted in Fig. 5A, the mRNA expression of EZH2 was significantly upregulated in SOX5-transfected MCF-7 cells, compared with vector-transfected cells, as demonstrated by RT-qPCR. This was further confirmed through western blotting. While in MDA-MB-231 cells, the knockdown of SOX5 resulted in significantly decreased EZH2 mRNA expression and markedly reduced EZH2 protein levels (Fig. 5B). These results indicated that SOX5 transactivated EZH2 expression. Notably, the knockdown of EZH2 was able 

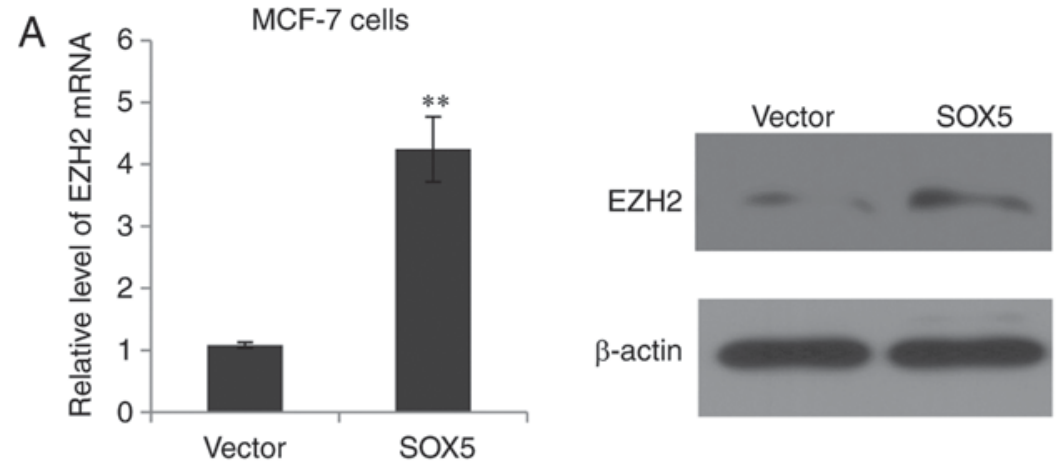

B

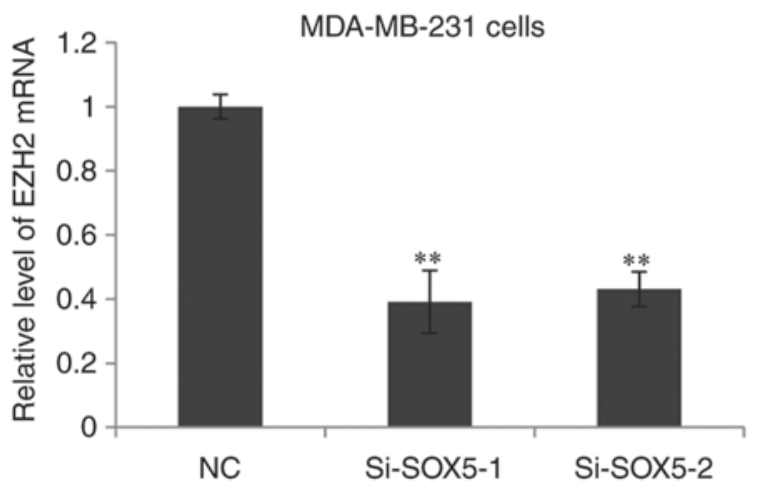

C

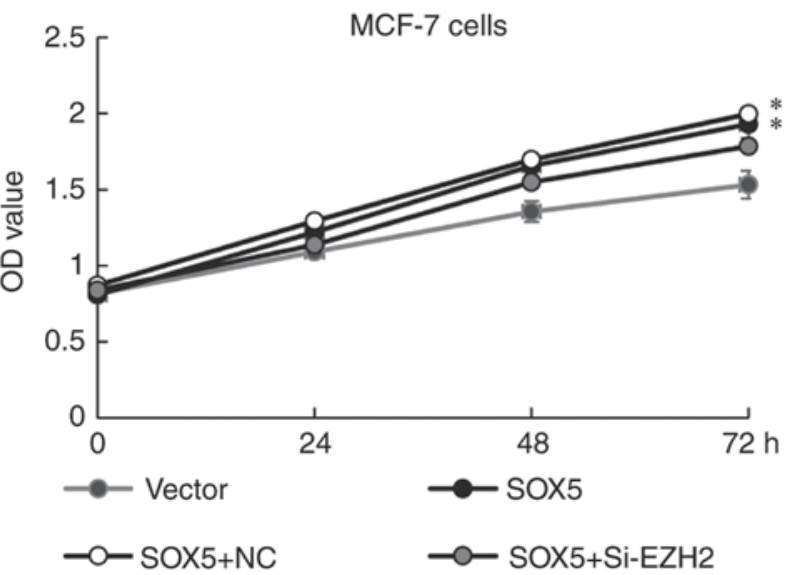

D
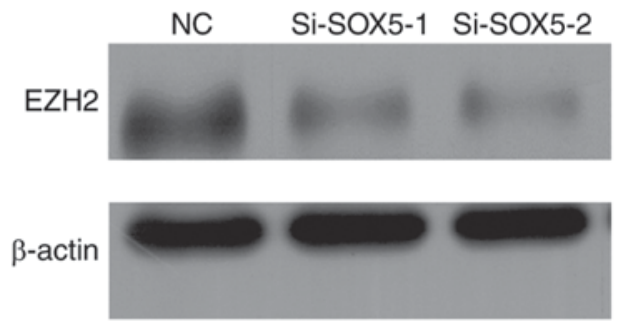

MDA-MB-231 cells

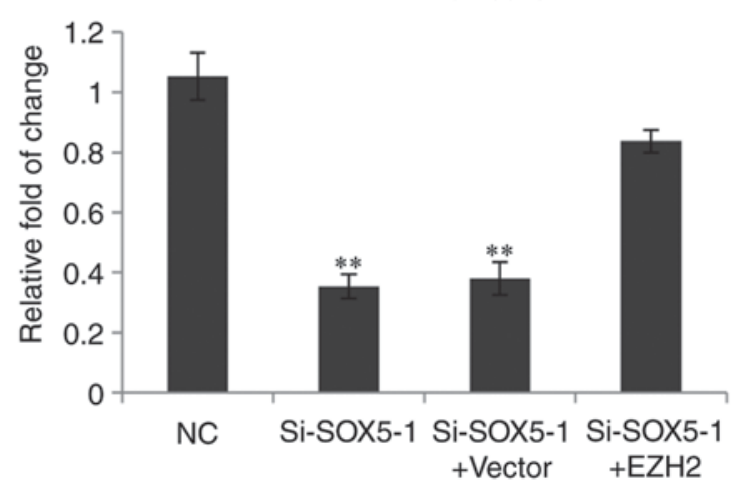

Figure 5. SOX5 induces breast cancer cell proliferation and invasion by modulation of EZH2. (A) RT-qPCR and western blotting was used to analyze EZH2 expression in SOX5- and vector-transfected MCF-7 cells. ${ }^{* *} \mathrm{P}<0.01$ vs. Vector. (B) RT-qPCR and western blotting was used to analyze EZH2 expression in MDA-MB-231 cells transfected with SOX5 siRNA (si-SOX5-1 and si-SOX5-2) or si-control. ${ }^{* *} \mathrm{P}<0.01$ vs. NC. (C) MCF-7 cells were transfected with empty vector or the SOX5 overexpression construct, SOX5 overexpression construct plus control siRNA or SOX5 overexpression construct plus si-EZH2, and the MTT assay was performed with the results being detected at $0,24,48$ and $72 \mathrm{~h}$. ${ }^{*} \mathrm{P}<0.05$ vs. Vector. (D) MDA-MB-231 cells were transfected with si-control, si-SOX5-1, si-SOX5-1 plus a vector or si-SOX5-1 plus EZH2, and a Matrigel assay was performed. The data are presented as the fold of change. ${ }^{* *} \mathrm{P}<0.01$ vs. NC. SOX5, sex determining region Y-box protein 5; NC, negative control; siRNA, small interfering RNA; EZH2, enhancer of zeste 2 polycomb repressive complex 2 subunit; RT-qPCR, reverse transcription-quantitative polymerase chain reaction; OD, optical density.

to overcome the SOX5 promoter effect on the proliferation of MCF-7 cells (Fig. 5C). Additionally, the invasion rate of MDA-MB-231 cells was significantly increased following treatment with EZH2, compared with the SOX5 knockdown group (Fig. 5D). These results indicated that SOX5 may regulate breast cancer cell proliferation and invasion through targeting EZH2 expression.

\section{Discussion}

Previously, a number of members of the SOX family, including SOX2 and SOX4, have been reported to be involved in tumor progression. SOX2 is a well-established stem cell regulator that is highly expressed in multiple tissue stem cells and sustain the infiltrative behavior in $\geq 25$ different cancer types, including cancers of the ovary, lung, skin, brain, breast, prostate and pancreas (19-21). Increased expression of SOX4 serves as an important role in human tumor development such as through regulating cell growth, invasion, EMT and apoptosis (22-25). However, the research regarding SOX 5 remains limited.

To the best of our knowledge, the present study is the first to demonstrate that SOX5 directly regulated EZH2 expression by transactivation, and thus promotes the proliferation and invasion of human breast cancer cells. Using ChIP-seq, qChIP and luciferase reporter assays, EZH2 was identified as a downstream target gene of SOX5. Using RT-qPCR and 
western blotting analysis, it was demonstrated that SOX5 regulates the expression of EZH2. The present data added to accumulating evidence regarding SOX family members being involved in breast cancer progression. As reported by Pei et al (14), SOX5 was overexpressed in highly invasive breast cancer cell lines, including MDA-MB-435 and MDA-MB-231 cells, and suppression of SOX5 expression inhibited the proliferation and migration of MDA-MB-231 cells. These data were consistent with the present study. In the present study, SOX5 was demonstrated to be frequently upregulated in breast cancer tissues compared with healthy breast tissue, and associated with a reduced overall survival rate, indicating that SOX 5 may serve as a poor prognostic biomarker in breast cancer. Additionally, the function of SOX5 was investigated in different cell lines, including MCF-7, the promotion of breast cancer cells proliferation and invasion indicated that SOX5 may be a potential oncogene. Notably, as reported by Tiwari et al (26), SOX4 directly regulated the expression of $\mathrm{EZH} 2$, and thus serves an indispensable role in EMT and cell survival in breast cancer (26). In patients with pancreatic cancer, the SOX4-EZH2 axis was demonstrated to be associated with the clinical outcome (27). Thus, we hypothesized that SOX4 and SOX5 may have a coordinated function on the EZH2 promoter to transactivate its expression. In the future, studies regarding the mechanistic association between SOX5 and EZH2 may be used for development of potential specifically-targeted therapies, and may benefit patients with breast cancer metastasis.

\section{Acknowledgements}

Not applicable.

\section{Funding}

No funding was received.

\section{Availability of data and materials}

The datasets used and-or analyzed during the current study are available from the corresponding author on reasonable request.

\section{Authors' contributions}

CS conceived and designed the study. YB provided technical assistance and performed the Transwell assay. YS and $\mathrm{ZZ}$ analyzed the data, KW performed the cell culture and wrote the paper. All authors read and approved the final manuscript.

\section{Ethics approval and consent to participate}

The present study was approved by the Research Ethics Committee of Weifang People's Hospital. All patients provided written informed consent.

\section{Patient consent for publication}

Not applicable.

\section{Competing interests}

The authors declare that they have no competing interests.

\section{References}

1. Siegel R, Ma J, Zou Z and Jemal A: Cancer statistics, 2014. CA Cancer J Clin 64: 9-29, 2014.

2. Bombonati A and Sgroi DC: The molecular pathology of breast cancer progression. J Pathol 223: 307-317, 2011

3. Hong $\mathrm{W}$ and Dong E: The past, present and future of breast cancer research in China. Cancer Lett 351: 1-5, 2014.

4. Torre LA, Bray F, Siegel RL, Ferlay J, Lortet-Tieulent J and Jemal A: Global cancer statistics, 2012. CA Cancer J Clin 65: 87-108, 2015

5. Lu J, Steeg PS, Price JE, Krishnamurthy S, Mani SA, Reuben J, Cristofanilli M, Dontu G, Bidaut L, Valero V, et al: Breast cancer metastasis: Challenges and opportunities. Cancer Res 69: 4951-4953, 2009.

6. Steeg PS: Targeting metastasis. Nat Rev Cancer 16: 201-218, 2016.

7. Martinez-Morales PL, Quiroga AC, Barbas JA and Morales AV: SOX5 controls cell cycle progression in neural progenitors by interfering with the WNT-beta-catenin pathway. EMBO Rep 11: 466-472, 2010.

8. Rescan PY and Ralliere C: A Sox5 gene is expressed in the myogenic lineage during trout embryonic development. Int J Dev Biol 54: 913-918, 2010.

9. Ma S, Chan YP, Woolcock B, Hu L, Wong KY, Ling MT, Bainbridge T, Webber D, Chan TH, Guan XY, et al: DNA fingerprinting tags novel altered chromosomal regions and identifies the involvement of SOX5 in the progression of prostate cancer. Int J Cancer 124: 2323-2332, 2009.

10. Chen Y, Liu W, Chao T, Zhang Y, Yan X, Gong Y, Qiang B, Yuan J, Sun M and Peng X: MicroRNA-21 down-regulates the expression of tumor suppressor PDCD4 in human glioblastoma cell T98G. Cancer Lett 272: 197-205, 2008.

11. Wang D, Han S, Wang X, Peng R and Li X: SOX5 promotes epithelial-mesenchymal transition and cell invasion via regulation of twist1 in hepatocellular carcinoma. Med Oncol 32: 461, 2015.

12. Zhang D and Liu S: SOX5 promotes epithelial-mesenchymal transition in osteosarcoma via regulation of Snail. J BUON 22: 258-264, 2017.

13. Huang DY, Lin YT, Jan PS, Hwang YC, Liang ST, Peng Y, Huang CY, Wu HC and Lin CT: Transcription factor SOX-5 enhances nasopharyngeal carcinoma progression by down-regulating SPARC gene expression. J Pathol 214: 445-455, 2008.

14. Pei XH, Lv XQ and Li HX: Sox5 induces epithelial to mesenchymal transition by transactivation of Twist 1 . Biochem Biophys Res Commun 446: 322-327, 2014.

15. Livak KJ and Schmittgen TD: Analysis of relative gene expression data using real-time quantitative PCR and the 2(-Delta Delta C(T)) method. Methods 25: 402-408, 2001.

16. Gonzalez ME, Moore HM, Li X, Toy KA, Huang W, Sabel MS, Kidwell KM and Kleer CG: EZH2 expands breast stem cells through activation of NOTCH1 signaling. Proc Natl Acad Sci USA 111: 3098-3103, 2014.

17. Kleer CG, Cao Q, Varambally S, Shen R, Ota I, Tomlins SA, Ghosh D, Sewalt RG, Otte AP, Hayes DF, et al: EZH2 is a marker of aggressive breast cancer and promotes neoplastic transformation of breast epithelial cells. Proc Natl Acad Sci USA 100: 11606-11611, 2003.

18. Truax AD, Thakkar M and Greer SF: Dysregulated recruitment of the histone methyltransferase EZH2 to the class II transactivator (CIITA) promoter IV in breast cancer cells. PLoS One 7: e36013, 2012.

19. Wuebben EL and Rizzino A: The dark side of SOX2: Cancer-a comprehensive overview. Oncotarget 8: 44917-44943, 2017.

20. Bulstrode H, Johnstone E, Marques-Torrejon MA, Ferguson KM, Bressan RB, Blin C, Grant V, Gogolok S, Gangoso E, Gagrica S, et al: Elevated FOXG1 and SOX2 in glioblastoma enforces neural stem cell identity through transcriptional control of cell cycle and epigenetic regulators. Genes Dev 31: 757-773, 2017.

21. Liu K, Xie F, Gao A, Zhang R, Zhang L, Xiao Z, Hu Q, Huang W, Huang Q, Lin B, et al: SOX2 regulates multiple malignant processes of breast cancer development through the SOX2-miR-181a-5p, miR-30e-5p-TUSC 3 axis. Mol Cancer 16: 62, 2017. 
22. Inoue $H$, Takahashi $H$, Hashimura $M$, Eshima $K$, Akiya $M$, Matsumoto T and Saegusa M: Cooperation of Sox4 with $\beta$-catenin-p300 complex in transcriptional regulation of the slug gene during divergent sarcomatous differentiation in uterine carcinosarcoma. BMC Cancer 16: 53, 2016.

23. Bilir B, Osunkoya AO, Wiles WG VI, Sannigrahi S, Lefebvre V, Metzger D, Spyropoulos DD, Martin WD and Moreno CS: SOX4 is essential for prostate tumorigenesis initiated by PTEN ablation. Cancer Res 76: 1112-1121, 2016.

24. Sun R, Jiang B, Qi H, Zhang X, Yang J, Duan J, Li Y and Li G: SOX4 contributes to the progression of cervical cancer and the resistance to the chemotherapeutic drug through ABCG2. Cell Death Dis 6: e1990, 2015

25. Yoon TM, Kim SA, Cho WS, Lee DH, Lee JK, Park YL, Lee KH, Lee JH, Kweon SS, Chung IJ, et al: SOX4 expression is associated with treatment failure and chemoradioresistance in oral squamous cell carcinoma. BMC Cancer 15: 888, 2015.
26. Tiwari N, Tiwari VK, Waldmeier L, Balwierz PJ, Arnold P, Pachkov M, Meyer-Schaller N, Schübeler D, van Nimwegen E and Christofori G: Sox4 is a master regulator of epithelial-mesenchymal transition by controlling Ezh2 expression and epigenetic reprogramming. Cancer Cell 23: 768-783, 2013.

27. Hasegawa S, Nagano H, Konno M, Eguchi H, Tomokuni A, Tomimaru Y, Asaoka T, Wada H, Hama N, Kawamoto K, et al: A crucial epithelial to mesenchymal transition regulator, Sox4-Ezh2 axis is closely related to the clinical outcome in pancreatic cancer patients. Int J Oncol 48: 145-152, 2016.

(c) (i) () ()

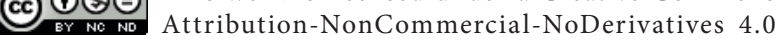
International (CC BY-NC-ND 4.0) License. 